\title{
Intermittent Communication Failure
}

National Cancer Institute

\section{Source}

National Cancer Institute. Intermittent Communication Failure. NCI Thesaurus. Code C133627.

Inconsistent or lack of intended communication of data among internal components or with other external devices. 